\title{
Physiological changes in a reaction time task: Further problems with Sokolov's dimension of stimulus "significance"
}

\author{
ROBERT J. BARRY \\ School of Education, University of New South Wales, Kensington 2033, New South Wales, Australia
}

\begin{abstract}
Sokolov placed attentional and response requirements in the same dimension of stimulus "significance," and proposed that these produced similar effects upon the magnitude and habituation rate of the OR. In a previous study, subjects were required to focus attention on stimulus events; here, the same stimuli served as imperative signals in a simple RT task. The effect of this manipulation upon several indices of the OR (GSR, respiration, HR, PPV, CPV, and EEG) failed to support Sokolov's position. The data obtained were used to further develop a hypothetical structure tentatively offered as a replacement of the unitary OR concept.
\end{abstract}

The orienting response (OR) of Sokolov (1963) may be described as a unitary response system sensitive to stimulus variation in three dimensions: novelty, intensity, and significance. Upon this response system has been built, by Sokolov and others, an elaborate theoretical structure concerned with the psychophysiological basis of perception and attentive processes. Barry $(1975,1976,1977 a, 1977 b)$ has critically examined the foundations of this theoretical structure by comparing Sokolov's detailed predictions regarding stimulus-response relationships with experimental findings from parametric investigations. These have centered upon six psychophysiological indices: electroencephalographic (EEG) alpha rhythm levels, galvanic skin response (GSR), heart rate (HR), respiration, and cephalic and peripheral pulse volumes (CPV and PPV, respectively). Changes in these measures have been investigated as a function of stimulus intensity (using auditory stimulation of from 20 to $50 \mathrm{~dB}$ SPL), novelty (by providing repeated cycles of the stimulus complex), and significance (indifferent stimuli vs. instructions to attend).

Barry (1977a) reported that the dependent variables listed above exhibited such markedly different behaviors in response to manipulation of stimulus intensity and novelty that the concept of a unitary OR appeared untenable: only two of the six measures examined covaried to the extent of having similar relationships with these two independent variables. It would thus appear that the simple OR concept which has been widely accepted in western psychophysiology may be an extreme oversimplification, having little empirical basis. Barry (1977b) varied stimulus "significance,"

Address requests for reprints to Robert J. Barry, School of Education, University of New. South Wales, Kensington 2033, New South Wales, Australia.
Sokolov's third stimulus dimension, by directing the attention of subjects to the stimulus complex. This attentional demand produced very small effects which were not as predicted by Sokolov; rather, they were able to be explained most parsimoniously in terms of a novelty reduction due to the experimental method used. [While the casual use of "attention" as a construct in that study cannot be used to denigrate attention as a meaningful stimulus attribute per se, its usage there was compatible with Sokolov (1963) and it can be used in that context to indicate the existence of problems with Sokolov's concept of "significance."'] That study did much to confirm the validity of the findings reported in Barry (1977a) with regard to the consistency of the stimulusresponse relationships obtained there. Together, those results add to the growing list of reports which cannot be encompassed within Sokolov's unitary OR theory.

Analysis of the results of those two studies suggested that the response measures listed above reflected the activity of at least three response systems involved in the perceptual process, rather than a simple unitary system. These were labeled in the following manner to allow ease of conceptualization, but it was stressed that the labels used represented summary statements rather than explanatory or functional devices:

Stimulus These responses occur at every stimulus register presentation regardless of intensity or novelty: $H R$ deceleration and $C P V$ dilation.

Intensity These responses reflect differences in register stimulus intensity: GSR and PPV constriction. 
Novelty These responses reflect differences in register the novelty of the stimulus complex: $G S R$, respiration pause, and EEG desynchronization.

This tentative conceptualization was offered in the hope of generating a new appraisal of the problems existing in this area of psychophysiology.

Sokolov (1963) included response requirements within his broad dimension of stimulus significance. Thus a stimulus attributed significance due to either attentional or response demand would be expected to elicit responses of greater magnitude with slower habituation; these effects should vary directly with the magnitude of the attributed significance. Barry (1977b) reviewed studies relevant to this point and noted contrary findings with the GSR and HR indicators of the OR when response effects were compared with those of attentional demand. With respiratory pause, CPV, PPV, and EEG indicators of the OR, insufficient data were available to allow definitive statements. Thus there is no body of evidence supporting the placement of attentional and response demand within the one dimension of stimulus parameters. The present study was directed at obtaining parametric data relevant to this problem for response requirements.

The paradigm selected for this involved the simple reaction time (RT) task without the use of a warning signal (in order to avoid anticipatory physiological responses). Teichner (1954) reviewed this field and concluded that a nonlinear relationship had been established between stimulus intensity and RT, with RT exhibiting a negatively accelerated decreasing function of intensity asymptotically approaching a physiological limit. At moderate intensities, such as is compatible with the OR, the speed of the relevant $\mathrm{RT}$ response could thus be expected to be a direct function of stimulus intensity, paralleling Sokolov's expected OR magnitude. Such a parallel would be consistent with the arousal aspect of the OR used in the relatively early learning studies of Maltzman and his colleagues (e.g., Maltzman \& Raskin, 1965) and, more recently, by Lodwig (1972). To date, such a relationship is more speculative than established.

\section{METHOD}

Twenty-four males, between 18 and 25 years of age and enrolled in an introductory psychology course at the University of Sydney, participated to fulfill a course requirement. This group was drawn from the same subject pool used in the previous study, and it matched that group on number, sex, and age range. Stimuli consisted of 2 -sec, $1,000-\mathrm{Hz}$ tones (presented binaurally via circumaural earphones), with an interstimulus interval randomly varying between 20 and $100 \mathrm{sec}$ with a mean of $60 \mathrm{sec}$. Each subject received a unique cycle of the stimulus intensities $20,30,40$, and $50 \mathrm{~dB}$ SPL (one of the 4 ! possible orders of the four intensities) effectively confounding individual differences and stimulus order. Calibration was carried out using an artificial ear coupler with a flat-plate adaptor manufactured at the University of Sydney. Eight cycles of the four stimuli were presented to enable habituation to be studied.

The GSR was recorded from lead electrodes on the volar surfaces of the first and second fingers of the subject's left hand and written out as ac-coupled resistance change on an E \& M Physiograph Six with a system time constant of $5 \mathrm{sec}$ and a paper speed of $2.5 \mathrm{~mm} / \mathrm{sec}$. Respiration was also recorded on the Physiograph from an $E \& M$ bellows pneumograph attached around the subject's chest. The EKG was recorded from EKG Lead 1 on a Grass IIID polygraph, with standard EKG time constant and paper speed of $15 \mathrm{~mm} / \mathrm{sec}$. PPV was recorded from an E \& M photoelectric pulse pickup attached to the distal volar surface of the third finger of the subject's left hand (which rested upon the arm of the subject's chair) and written out on the Physiograph. Another pulse pickup was placed against the zygoma, where a pulse could be manually felt. This was held in position by the earphones, and its output was recorded on the Physiograph as the CPV. The electroculargram (EOG) was recorded on the Grass polygraph and used to check that the subject's eyes were open as requested; it will not be discussed further. The subjects were fitted with silver/silver chloride pad electrodes at $\mathrm{O}_{1}, \mathrm{P}_{3}$, and $\mathrm{C}_{\mathrm{z}}$ (Jasper, 1958). $\mathrm{C}_{\mathrm{z}}$ was grounded and the activity between $\mathrm{O}_{1}-\mathrm{P}_{3}$ recorded on the Grass polygraph as the occipital EEG. Stimulus events were simultaneously indicated on a separate channel of each recording machine through the stimuluspresentation system. Subjects sat in a comfortable armchair in the dark with their eyes open in a sound-reduced light-tight electrically shielded room.

After application of recording devices as outlined above, the subjects were introduced to a trigger-like switch with a "pistol-grip" built into the subject chair. This was operated by the subject's right hand. At least 10 practice trials with a $40-\mathrm{dB} 600-\mathrm{Hz}$ tone as the imperative RT signal were used to accustom the subject to the RT procedure. Each subject was required to reach a criterion of three successive RTs of less than $300 \mathrm{msec}$; two subjects required 11 trials and two required 12 trials to reach this criterion. The subject was then instructed that a range of tones would be used as the RT signal and that he was to respond as quickly as possible to each stimulus. RT was read off a BOTH chronoscope and recorded.

Apart from changes necessitated by the RT paradigm, this procedure replicated that used in Barry (1977b) to'investigate attentional demand effects, where the subjects were instructed to attend to the stimulus sequence in preparation for a postexperimental enquiry. Essentially, the stimuli subjects were asked to attend to in Barry (1977b) now served as imperative RT stimuli. The experimental analysis reported here is thus based on a threeway design (over response requirements, stimulus intensity, and novelty), with repeated measures on the last two variables.

\section{RESULTS}

RT and each physiological variable will be discussed in turn to examine the effects of stimulus intensity and novelty on these measures in the context of response demand. A formal comparison of the responses found in this study with those found in the last will be used to extend the present knowledge of these effects and the knowledge available on response demand as an independent variable.

It was felt essential to ensure that the responses measured in this experiment were valid estimators of the activity in each psychophysiological channel. The introduction of response requirements in the form of 
the RT paradigm could conceivably have altered the nature of the response in some cases, e.g., HR acceleration was tentatively expected here rather than the HR deceleration previously found. Accordingly, with those variables in which the nature of the response could change in this manner (HR, CPV, and PPV), the response type was investigated. The methods used to evaluate the EEG and respiratory responses (percent change in activity) would automatically reflect any such change in response type (e.g., enhanced alpha activity would yield a negative change score if the previous criteria were applied). Finally, the GSR, as herein defined, can occur only in a single response type, so the use of the existing response criteria was acceptable.

\section{RT}

Mean values of RT in milliseconds are shown as a function of stimulus intensity in Figure 1 and as a function of stimulus repetition in Figure 2. There
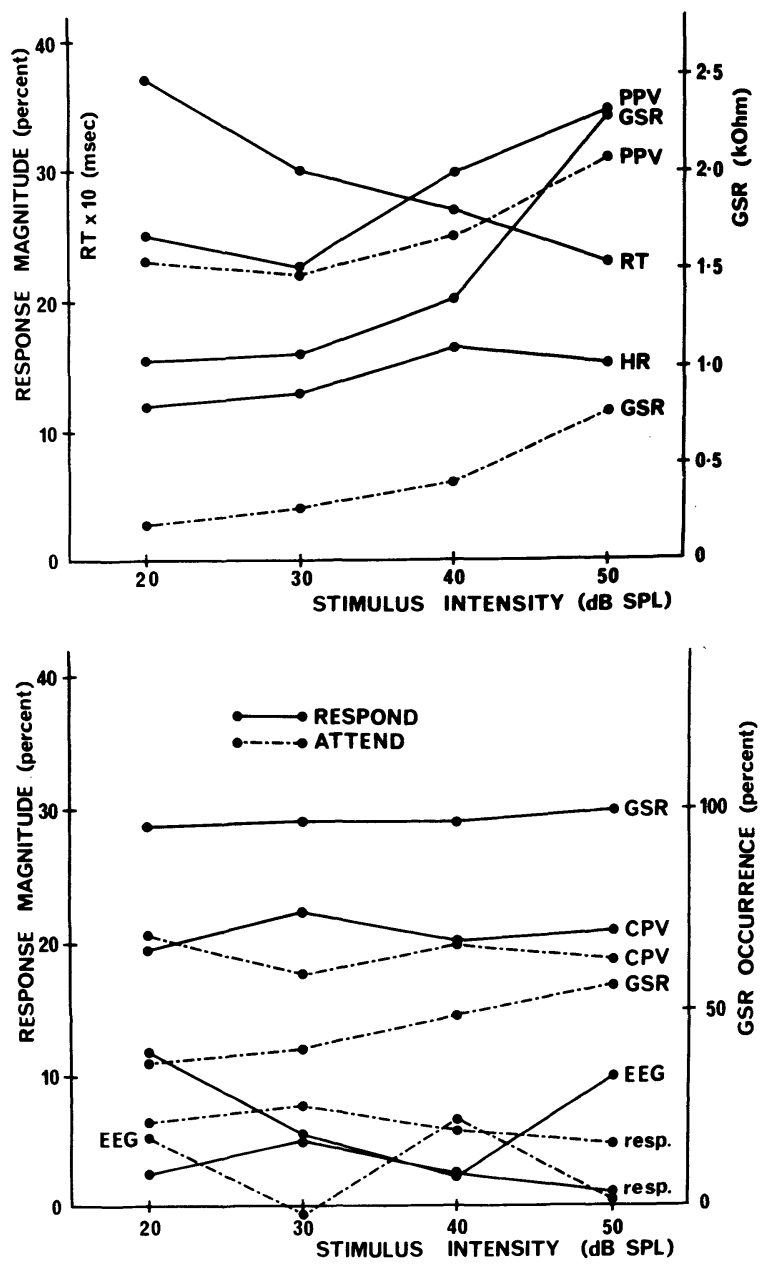

Figure 1. Response magnitude (percent change from prestimulus baseline) as a function of stimulus intensity. $R T$ and response occurrence of the GSR are also included. Top: measures showing significant intensity effects. Bottom: measures not affected by stimulus intensity.
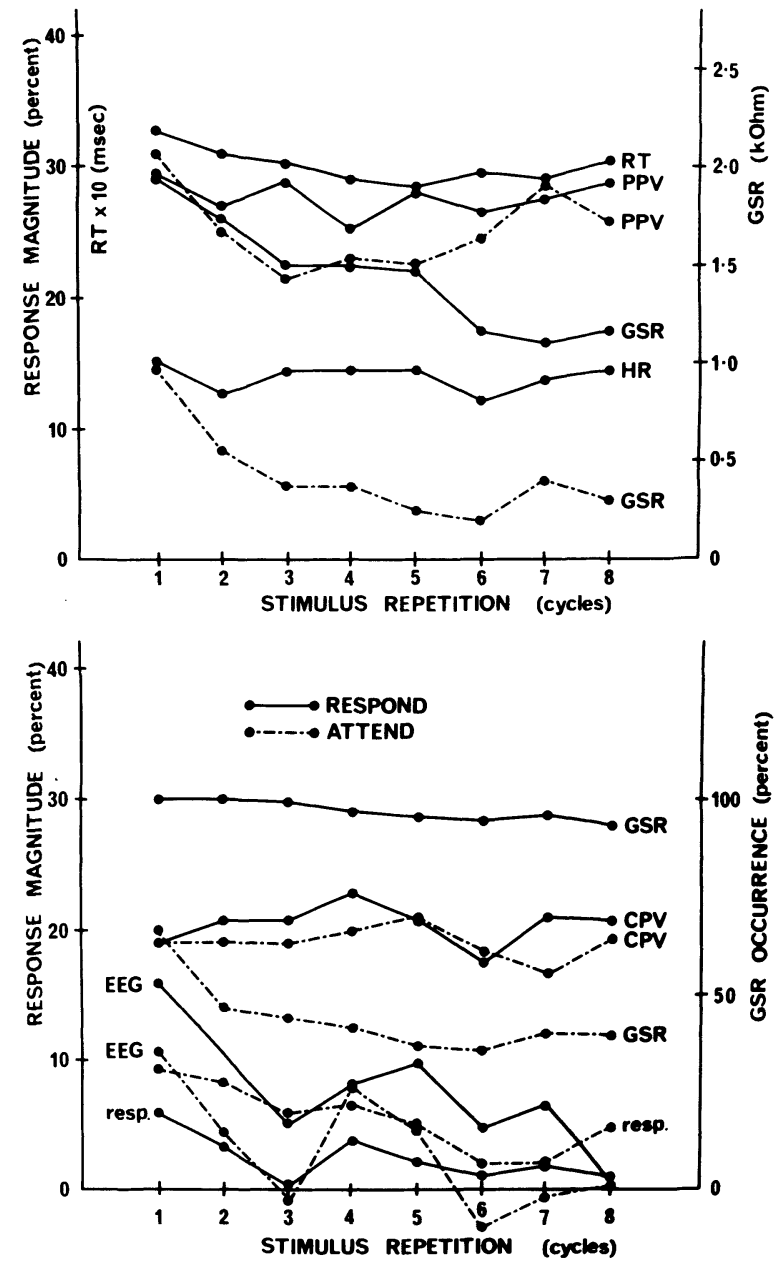

Figure 2. Response magnitude (percent change from prestimulus baseline) as a function of decreasing stimulus novelty. RT and response occurrence of the GSR are also shown. Top: measures which showed intensity effects. Bottom: measures not significantly affected by stimulus intensity.

was a stimulus intensity effect, significant well beyond the .05 level, mainly describable by a linear trend $[\mathrm{F}(1,69)=327.8, \mathrm{MSe}=6,436.2]$ with a quadratic trend component $[\mathrm{F}(1,69)=11.8]$ also present. There was a significant quadratic trend over repeated cycles $[F(1,23)=14.84$, MSe $=4,834.6]$, but this could not be construed as indicating habituation. There was a significant interaction between the linear trend over repeated cycles and stimulus intensity $[\mathrm{F}(3,69)=5.18, \mathrm{MSe}=5,421.7]$, as indicated in Figure 4. This appears, by inspection, to be due to a reduction in $\mathrm{RT}$ with stimulus repetition for the 20-dB stimulus. Such an improvement in performance cannot be related to the concept of habituation; rather, the initial uncertainty of subjects regarding the onset of such a low-level stimulus (particularly in relation to the 40-dB training signal) seems plausible. These results confirm the traditional findings of a large stimulus intensity effect with a withinsubjects design. The remarkably consistent level of 
responding throughout the experiment indicates that a consistent level of response demand was maintained. This is most important in considering the behavior of the response systems discussed below.

\section{GSR}

The GSR was defined as a measurable drop in resistance with latency 1 to $5 \mathrm{sec}$ following stimulus onset. Mean values of response amplitude are plotted against stimulus intensity in Figure 1 and against repeated stimulus cycles in Figure 2 (top graphs). Following the procedure of the previous experiments, a Friedman analysis of variance by ranks was carried out on the scores averaged over trials and (separately) on the scores averaged over intensity. These showed significant stimulus intensity $\left(\chi_{\mathrm{r}}^{2}=51.09, \mathrm{df}=3\right)$ and trials $\left(\chi_{\mathrm{r}}^{2}=54.92, \mathrm{df}=7\right)$ effects, the latter being interpretable as reflecting habituation of the GSR. Figures 1 and 2 also show the results obtained previously under "attend" conditions. Comparison of the two sets of data suggests that the response demand of this study has produced an increased response magnitude at all data points. A MannWhitney U-test comparing the average response over all stimulus presentations between subjects in the two experiments $\left(\mathrm{U}=521.0, \mathrm{n}_{1}=\mathrm{n}_{2}=24\right)$ confirmed this enhancement. The frequency of response occurrence was extracted from the data and was found to range from $96 \%$ to $100 \%$ in a nonsystematic manner over intensity and repetition. These data are graphed in Figures 1 and 2 (bottom graphs). This was a much higher level of response occurrence than found previously. There was a significant difference in the frequencies of occurrence as a function of intensity $\left(\chi_{3}^{2}=520.3\right)$, and this remained when allowance was made for the increased level of responding by adjusting the expected frequencies in the chi-squared analysis $\left(\chi_{3}^{2}=20.6\right)$. This finding indicates that there is not the dependence of response occurrence upon stimulus intensity found in the previous experiment. Similar findings were obtained with response occurrence as a function of repetition of the stimulus complex: a significant difference overall $\left(\chi_{7}^{2}=517.6\right)$ remained after expected values were adjusted to equate the mean level of responding $\left(\chi_{7}^{2}=21.1\right)$. This last finding indicates that response emission did not decrease with repetition as had happened previously. Thus, response demand in this experiment has been associated with enhanced GSR activity reflected in both a larger response amplitude which still showed habituation and in the continued emission of responses throughout the duration of the experiment; this last is in marked contrast to previous results. Thus, the GSR has been shown to be sensitive to response requirements in more or less the fashion predicted by Sokolov to occur with "significance" produced by either attentional or response requirements.

\section{Respiration}

The periods of the respiratory cycle immediately before stimulus onset and that containing the stimulus onset were scored from the record. The difference between these was expressed as a percentage increase in respiratory period. Mean values of this response measure, together with corresponding information from the previous study, are shown in Figures 1 and 2. These data were subjected to a three-way analysis of variance over response demand, intensity, and repetition, with repeated-measures trend analyses on the last two variables. There was some reduction in magnitude with the increase in response demand, but this was of borderline significance $[\mathrm{F}(1,46)=3.94$, MSe $=1,061.2$, cf. critical $\mathrm{F}$ at $\mathrm{p}=.05$ is 4.06]. There was a significant linear reduction of response magnitude with increasing stimulus intensity $[F(1,138)=$ 4.13, $\mathrm{MSe}=251.6]$ and a significant linear trend over repeated stimuli $[\mathrm{F}(1,322)=10.25$, MSe $=$ 311.5]. This last supports the findings of habituation previously reported.

\section{HR}

The nature of the HR response was investigated by scoring individual protocols for the R-R intervals of 3 prestimulus and 9 poststimulus beats associated with the first and last presentations of the 20- and 50-dB stimuli. Mean HRs from these 96 protocols were obtained at each beat and are displayed in Figure 3. This indicated that the $H R$ response obtained in this experiment was one of acceleration rather than the deceleration previously reported under attend conditions. These responses were compared by examining the quadratic trends over the 10 beats from Prestimulus Beat 1 to Poststimulus Beat 9. There was a significant interaction between this quadratic trend and group $[\mathrm{F}(1,46)=32.14$,

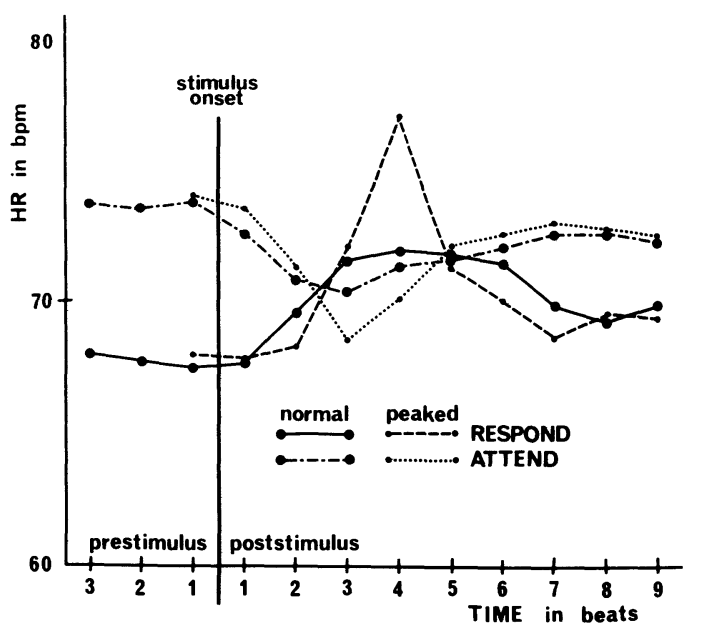

Figure 3. Mean $H R$ response from the first and last presentation of the 20- and 50-dB stimuli under both "attend" and "respond" conditions. "Peaked" responses corrected for latency difference are also shown. 
MSe $=63.6]$, indicating that the direction of the HR change differed significantly between the groups, and a marked difference in prestimulus HR level [ $t(190)$ $=7.94]$.

Barry (1977a) discussed the conceptual problem of latency in the context of the HR response, and proposed a procedure aimed at generating the form of the HR response corrected for differences in latency between individual responses. This was applied to the present HR response. The beat of maximum HR (cf. minimum HR under "attend" conditions) within the first 6 poststimulus beats in each of these protocols was labeled P. Mean HRs over Beats $P$, $\mathrm{P}-1, \mathrm{P}-2, \mathrm{P}-3, \mathrm{P}-4, \mathrm{P}+1, \mathrm{P}+2, \mathrm{P}+3, \mathrm{P}+4$, and $P+5$ were then calculated and are plotted in Figure 3 as the "peaked response." This shows a latency-corrected response which appears as a simple fleeting acceleration of about $13 \%$ of the prestimulus HR with a latency (measured to the response peak) of about 4 beats. This is an obviously different form of response from the deceleratory response found under attentional-demand conditions and precludes a statistical between-study analysis of the type carried out with the respiratory response.

The HR response was thus defined as the largest percentage increase in $\mathrm{HR}$, relative to Prestimulus Beat 1 , occurring in the first 6 poststimulus beats. Mean values of this response are displayed in Figures 1 and 2 . A two-way repeated measures trend analysis indicated a significant linear trend over stimulus intensity $[\mathrm{F}(1,69)=13.0, \mathrm{MSe}=116.4]$ but no significant trend over repeated stimulus presentations. There was, however, a significant interaction between linear trend over repeated cycles and stimulus intensity $[\mathrm{F}(3,69)=3.7, \mathrm{MSe}=66.9]$. By inspection of Figure 4, this appeared to be due to an increasing

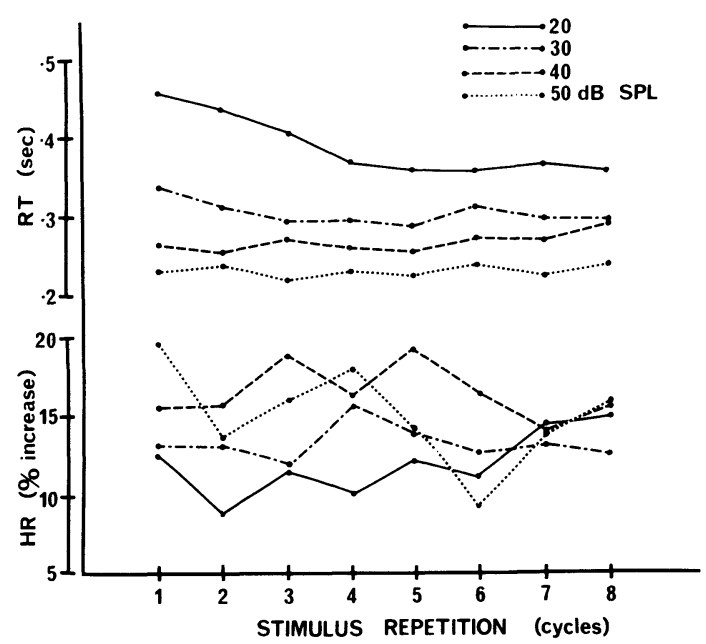

Figure 4. RT and HR response magnitude as a function of decreasing stimulus novelty. Results are shown separately for each stimulus intensity to indicate Intensity by Trial interactions. response magnitude associated with repetition of the $20-\mathrm{dB}$ stimulus. This is very similar to the effect found with RT; it is clearly independent of the concept of habituation.

\section{PPV}

The form of the PPV response was checked against that previously obtained under attentional demand conditions and found to be virtually identical: a slow reduction in amplitude (vasoconstriction) bottomingout at about Beat 9 poststimulus. The smallest pulse amplitude occurring between 7 and 12 beats poststimulus was taken as the point of maximum response, as in the previous study. The difference between this amplitude and that at Prestimulus Beat 1 was converted to a percentage decrease, and mean values of this response are shown as a function of intensity in Figure 1 and as a function of repetition in Figure 2. A three-way ANOVA, as used with respiration, indicated a significant stimulus intensity effect appearing in both linear $[\mathrm{F}(1,138)=48.52, \mathrm{MSe}=374.4]$ and quadratic $[F(1,138)=11.98]$ trends over intensity. There was also a singificant quadratic trend over repeated presentations of the stimulus complex $[\mathrm{F}(1,322)=11.16, \mathrm{MSe}=296.2]$, which could not be conceptualized as simple habituation. There was no significant between-subjects effect of response requirement, and no other significant effects.

\section{CPV}

As with PPV, the CPV response was found to be of the same form as that obtained under attentionaldemand conditions: a simple momentary dilation of the superficial temporal artery occurring with maximum effect at approximately 3 beats poststimulus. Accordingly, individual protocols were scored for the maximum increase in CPV (relative to Prestimulus Beat 1) occurring within the first 6 beats poststimulus. Mean values of this response are included in Figures 1 and 2. A three-way ANOVA over response requirements, stimulus intensity, and repetition indicated that there were no significant main effects or interactions with this measure.

\section{EEG}

The amplitude of occipital alpha activity was hand-scored for the 1-sec intervals before and after each stimulus onset, as described in Barry (1976, 1977b) and Barry and Beh (1972, 1976). The percentage change in alpha activity was taken as the response measure and is displayed against intensity and stimulus repetition in Figures 1 and 2. These data were submitted to a three-way ANOVA as described above. This indicated a significant quadratic trend over intensity $[\mathrm{F}(1,138)=4.14, \mathrm{MSe}=938.1]$ with significant interactions showing different quadratic $[\mathrm{F}(1,138)=3.98]$ and cubic $[\mathrm{F}(1,138)=5.17]$ 
trends between the groups differing in response demand. These differences are difficult to conceptualize and will be discussed later. There was a clear habituation effect shown by a significant linear trend over stimulus cycles $[\mathrm{F}(1,322)=13.78, \mathrm{MSe}=$ $1,071.5]$, with no other significant main effects or interactions.

\section{DISCUSSION}

It must be remembered that the reaction time paradigm used in this study to investigate the physiological outcome of response demand may also have varied the level of attention exhibited by subjects relative to that attained in Barry (1977b), as well as the novelty of the stimulus complex. This would be impossible to estimate directly, but care must be taken in regard to the labeling of effects as due purely to response demand, particularly in the case of those variables previously shown to be sensitive to novelty. The measure of response demand used here was the actual RT obtained at each stimulus presentation, and this showed no overall decline in performance level with repeated presentation of the stimulus complex. It can thus be concluded that a sustained level of response demand was achieved and that the effects of this, if they exist in the response systems sampled here, should be apparent over all stimulus presentations.

The GSR exhibited markedly different stimulusresponse relationships in this study compared with previous findings, and these appear to be compatible with this expectation of a continuing response-demand effect. In previous studies, both the number of GSRs and the mean amplitude of the GSR decreased with stimulus repetition in such a way as to support the contention that the GSR was sensitive to novelty. In the present study, response amplitude was enhanced but still habituated in accordance with the operation of this variable, while response occurrence was maintained at a high level throughout. This indicates that the GSR is quite sensitive to response demand in addition to its sensitivity to novelty, and in this respect it behaves as predicted by Sokolov. The respiratory response also appeared to be modified to some extent in this experiment, exhibiting a nonsignificant reduction in magnitude compared with that obtained under "attend" conditions. This might be attributed to either a direct effect of response demand or to a reduction in stimulus novelty produced by the RT-training procedure, even though a different tone was used in this part of the experiment. The latter explanation is supported by the lack of a continuing effect over trials: response demand remained fairly consistent throughout (as evidenced by RTs), but the difference in respiratory response appears to decrease with stimulus repetition (see Figure 2), as would be expected if the response system responded primarily to novelty.

When we consider HR, we find the greatest change produced by response demand: the cardiac system has been found to generate an entirely new form of response, one of acceleration of the HR. This system produced a response of HR deceleration under the nonenergetic conditions of the previous studies. This deceleratory response was found not to be sensitive to stimulus intensity, stimulus novelty, or attentional demand. Under the conditions of the RT paradigm, the cardiac system exhibited a response of HR acceleration which was sensitive to stimulus intensity and which showed very similar behavior with stimulus variation to that shown by RT itself. Together with the difference in latencies of the two responses, these differences in response determinants indicate substantial differences in underlying mechanism.

The marked reduction in prestimulus HR level obtained in the "respond" condition when compared with that of the previous "attend" condition is also of some interest. The paradigm of the unwarned RT task with variable ISI as used here was expected to eliminate the anticipatory HR deceleration found in most RT studies (see Lacey \& Lacey, 1978, for a recent review). Indeed, Figure 3 indicates support for this expectation with the absence of marked prestimulus deceleration. However, the difference in prestimulus level between the two conditions indicates some effect of response demand, consistent with those tonic changes in HR conceptualized by the Laceys as reflecting situational stereotypy (see, for example, Lacey, Kagan, Lacey, \& Moss, 1963). The role of such tonic changes in elicitation of the phasic HR response is beyond the scope of the present report. However, if such changes are a necessary concomitant of response demand, then the HR component of the OR should still behave as conceptualized in Sokolov's unitary OR theory if his generalization of "significance" to response conditions is to be supported.

PPV continued to show the same vasoconstrictive response as found in the previous studies, and, although reflecting stimulus intensity, is apparently insensitive to response requirements. CPV also behaved as in the previous studies, failing to show any effect of stimulus intensity or repetition, and appears to be insensitive to response demand. The amplitude of EEG alpha activity showed significant differences in quadratic and cubic trends over stimulus intensity as a function of response demand. It appears impossible to causally relate such interactions in complex trends to a simple response requirement, and it is postulated here that such interactions reflect the attainment of statistical significance rather than psychologically meaningful findings. The lack 
of a consistent intensity effect will thus be taken as indicating the insensitivity of the EEG to stimulus intensity in this range. The EEG showed significant habituation with stimulus repetition; there were no other effects attributable to response demand.

Combination of the data from the present series of experiments regarding the influence of the four independent variables studied upon the magnitude of the physiological responses found here is summarized in Table 1. This account allows the extension of the schema outlined in Barry (1977b) as a possible replacement for Sokolov's unitary OR system. The addition of a response category to handle the information generated in the present study expands the schema as shown below.

Stimulus These responses occur at every stimulus register presentation regardless of intensity or novelty: $H R$ deceleration and $C P V$ dilation.

Intensity These responses reflect differences in register stimulus intensity: GSR, HR acceleration, and PPV constriction.

Novelty These responses reflect differences in register the novelty of the stimulus complex: $G S R$, respiratory pause, and EEG alpha desynchronization.

Response These responses reflect response deregister mand: GSR and HR acceleration.

It should be noted that this is a tentative systematization of the mass of data generated in these experiments, and must be viewed in the context of the reservations and provisos discussed in its development.

It is apparent from a consideration of these results that attention and response requirements cannot be placed on the single dimension of "significance" proposed by Sokolov. Further, we have seen an upward expansion from Sokolov's unitary OR concept (in which all the physiological variables considered here covaried) to a fourfold conceptualization in which the physiological responses form four independent subgroups with internal covariation. This new systematization offers the most parsimonious conceptualization of the data gathered in this series of studies. In essence, these experiments fail to support the concept of the OR as developed by Sokolov and, in its place, offer a much more complex structure to cover the psychophysiological responses to simple low-level stimuli. It should be recognized that this complex schema is founded on mean data-
Table 1

Sensitivities of the Physiological Measures Used Here to the Independent Variables Manipulated

\begin{tabular}{llccc}
\hline & \multicolumn{4}{c}{ Independent Variables } \\
\cline { 2 - 5 } $\begin{array}{c}\text { Dependent } \\
\text { Variable }\end{array}$ & $\begin{array}{c}\text { Inten- } \\
\text { sity }\end{array}$ & $\begin{array}{c}\text { Novel- } \\
\text { ty }\end{array}$ & $\begin{array}{c}\text { Atten- } \\
\text { tion }\end{array}$ & $\begin{array}{c}\text { Re- } \\
\text { sponse }\end{array}$ \\
\hline GSR & Yes & Yes & No & Yes \\
Respiration & No & Yes & No & No \\
HR Deceleration & No & No & No & $*$ \\
HR Acceleration & Yes & No & $*$ & Yes \\
PPV & Yes & No & No & No \\
CPV & No & No & No & No \\
EEG & No & Yes & No & No \\
\hline
\end{tabular}

*Not applicable.

it is, in fact, a conceptualization which encompasses large-scale effects on sample means and takes no account of possible complexities introduced by individual differences. Further work in this area is in preparation.

\section{REFERENCES}

BARRY, R. J. Low-intensity auditory stimulation and the GSR orienting response. Physiological Psychology, 1975, 3, 98-100.

BARRY, R. J. Failure to find the "local" EEG OR to low-level auditory stimulation. Physiological Psychology, 1976, 4, 171-174.

BARRY, R. J. Failure to find evidence of the unitary OR concept with indifferent low-intensity auditory stimuli. Physiological Psychology, 1977, 5, 89-96. (a)

BARRY, R. J. The effect of "significance" upon indices of Sokolov's orienting response: A new conceptualisation to replace the OR. Physiological Psychology, 1977, 5, 209-214. (b)

BARRY, R. J., \& BEH, H. C. EEG desynchronization as a function of intensity of visual stimulation. Psychonomic Science, 1972, 26, 241-242.

BARRY, R. J., \& BEH, H. C. EEG correlates of the afterimage of visual stimulation. Psychophysiology, 1976, 13, 75-80.

JASPER, H. H. Report of the committee on methods of clinical examination in electroencephalography. Electroencephalography and Clinical Neurophysiology, 1958, 10, 370-375.

LACEY, B. C., \& LACEY, J. I. Two-way communication between the heart and the brain. American Psychologist, 1978, 33, 99-113.

LACEY, J. I., KAGAN, J., LACEY, B. C., \& Moss, H. A. The visceral level: Situational determinants and behavioral correlates of autonomic response patterns. In P. H. Knapp (Ed.), Expression of the emotions in man. New York: International Universities Press, 1963.

LoDwIG, A. K. Experimental manipulation of orienting reflex during semantic conditioning. Journal of Experimental Psychology, 1972, 96, 416-424.

Maltzman, I., \& Raskin, D. D. Effects of individual differences in the OR on conditioning and complex processes. Journal of Experimental Research in Personality, 1965, 1, 1-16.

Sokolov, E. N. Perception and the conditioned reflex. Oxford: Pergamon Press, 1963.

Teichner, W. H. Recent studies of simple reaction time. Psychological Bulletin, 1954, 51, 128-149.

(Received for publication August 9, 1977; revision accepted September 18, 1978.) 